\title{
Stents com Everolimus: Resultados do Programa SPIRIT. Análise dos Estudos Fundamentais com o Stent Farmacológico Xience $^{\mathrm{TM}} \mathrm{V}$
}

\author{
José de Ribamar Costa Jr. ${ }^{1}$, Dimytri Siqueira ${ }^{1}$, Alexandre Abizaid ${ }^{1}$
}

\section{RESUMO}

Recentemente aprovado para uso clínico pelas principais agências reguladoras mundiais, incluindo a Agência Nacional de Vigilância Sanitária (ANVISA) e o Food and Drug Administration (FDA), o stent farmacológico de segunda geração Xience ${ }^{\mathrm{TM}}$ (Abbott Laboratories, Abbott Park, Illinois, Estados Unidos) combina uma plataforma de cromo-cobalto de baixo perfil a uma droga antiproliferativa potente, o everolimus (análogo do sirolimus), carreada por meio de um polímero durável, bastante biocompatível. Nesta revisão, sintetizamos os resultados dos principais ensaios clínicos controlados que culminaram com a aprovação do Xience ${ }^{\mathrm{TM}}$ para uso clínico, bem como apresentamos os principais estudos clínicos que estão em andamento envolvendo esse novo stent farmacológico.

DESCRITORES: Stents farmacológicos. Sirolimo/análogos \& derivados. Ensaios controlados aleatórios.

\begin{abstract}
SUMMARY
Everolimus-eluting Stents: Results of the SPIRIT

Trial. A Critical Appraisal of the Pivotal Studies with the New Xience ${ }^{\mathrm{TM}} \mathrm{V}$ Stent

Recently approved worldwide by regulatory agencies (including ANVISA and FDA), the $2^{\text {nd }}$ generation drug-eluting stent (DES) Xience ${ }^{T M}$ (Abbott Laboratories, Abbott Park, IL, USA) combines an ultra-thin cobalt-chromium platform and a potent sirolimus-analog anti-proliferative drug, everolimus, carried by a biocompatible durable polymer. In the present review, the authors summarize the main findings of the preliminary randomized clinical trials that resulted in the approval of this new DES for clinical use and present the next studies planned with the Xience ${ }^{T M}$ stent.
\end{abstract}

DESCRIPTORS: Drug-eluting stents. Sirolimus/analogs \& derivatives. Randomized controlled trial.

muito tardia (após o primeiro ano da intervenção), especialmente após a descontinuidade da terapia antiplaquetária dupla ${ }^{5-7}$.

Análises histológicas em modelos ex vivo e com métodos de imagem intracoronária (ultra-som, tomografia óptica, etc.) in vivo atribuíram parte desses efeitos adversos à presença do polímero durável utilizado por esses SF para carrear o fármaco antiproliferativo ao local tratado ${ }^{8-10}$.

Visando à manutenção da marcante supressão da resposta neo-intimal e às baixas taxas de reestenose angiográfica e clínica, e, ao mesmo tempo, incrementar a flexibilidade, a radiopacidade, a resistência à fratura e, sobretudo, a segurança (com uso se polímeros mais biocompatíveis ou mesmo bioabsorvíveis) desses novos dispositivos, as pesquisas na cardiologia intervencionista moveram seu foco para o desenvolvimento da chamada segunda geração de SF, da qual fazem parte o stent Endeavor ${ }^{\mathrm{TM}}$ (Medtronic, Mineapolis, Minnesota, Estados Unidos) e o Xience ${ }^{\mathrm{TM}} \mathrm{V}$ (Abbott Laboratories, Abbott Park, Illinois, Estados Unidos). 
A seguir, discutiremos com brevidade os elementos que compõem o stent $\mathrm{Xience}^{\mathrm{TM}} \mathrm{V}$ e apresentaremos os resultados dos principais estudos controlados (SPIRIT I, II e III), que culminaram com a aprovação desse novo instrumental pelas principais agências reguladoras mundiais.

\section{STENT FARMACOLÓGICO XIENCETM V}

Esse SF combina uma plataforma ultrafina e bastante flexível (Multi-Link Vision), recoberta por um polímero biocompatível (de flúor), contendo um potente fármaco antiproliferativo, o everolimus (Figura 1). A seguir, detalharemos cada um dos elementos essenciais que compõem esse novo SF:

\section{Stent Multi-Link Vision ${ }^{\mathrm{TM}}$ (Guidant Corporation, Indianapolis, Indiana, Estados Unidos)}

Essa plataforma de cromo-cobalto (Cro-Co L-605) com hastes ultrafinas $\left(0,0032^{\prime \prime}\right.$ ou $\left.81 \mu \mathrm{m}\right)$ foi desenhada para garantir excelente navegabilidade e flexibilida$\mathrm{de}^{11}$, permitindo alcançar regiões bastante tortuosas e calcificadas. Outra potencial vantagem do Cro-Co diz respeito a sua maior radiopacidade, facilitando com isso a pós-dilatação do stent implantado.

Nos diâmetros de 2,5 mm e 3,0 mm, esse stent possui seis células, ao passo que os diâmetros de $3,5 \mathrm{~mm}$ e 4,0 $\mathrm{mm}$ apresentam nove células, a fim de aumentar a superfície de contato do stent com o endotélio e, com isso, otimizar a concentração e a distribuição do fármaco antiproliferativo.

Essa plataforma é montada sobre um balão de Pebax de baixo perfil (5 F).

\section{Everolimus (Certican, Novartis, Basiléia, Suíça)}

Esse fármaco é análogo ao sirolimus e tem sido usado sobretudo na prevenção de rejeição após transplantes.

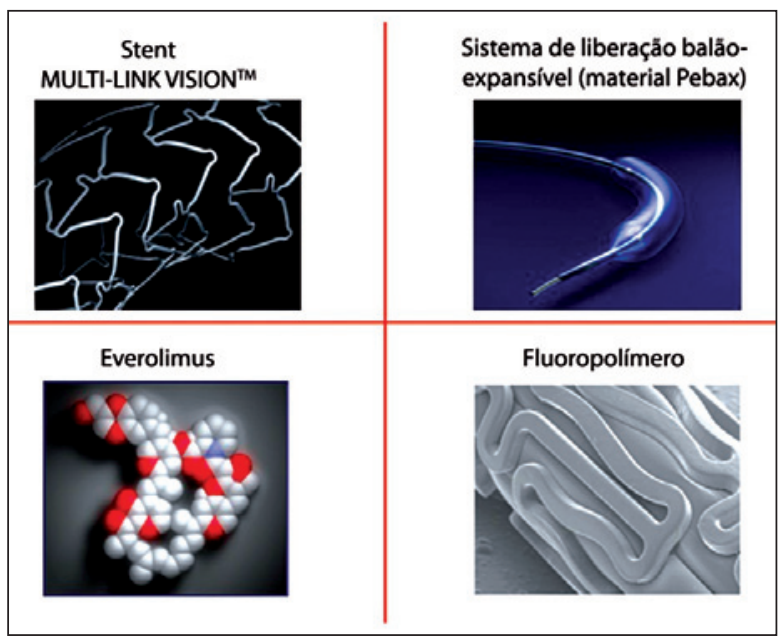

Figura 1 - Principais componentes do stent farmacológico de segunda geração $X_{\text {ience }}^{T M} \mathrm{~V}$.
Para exercer suas atividades antiproliferativas, o everolimus se liga a proteínas citosólicas específicas, as imunofilinas. O complexo formado se une a uma proteína específica do ciclo celular, a mTOR (mamalian Target of Rapamycin), e inibe sua ativação. Essa ação é mediada por um receptor intracelular, a proteína FK 506 (FKBP 12), formando o complexo everolimus-FKBP ${ }^{12}$. A inibição da mTOR impede a proliferação de células $\mathrm{T}$ e, com isso, bloqueia a passagem do ciclo celular da fase G1 para a S.

Mais recentemente, Verheye et al. ${ }^{13}$ demonstraram uma nova e única propriedade do everolimus em modelos experimentais. Estudando coelhos com doença aterosclerótica coronária estabelecida, esse grupo de pesquisadores evidenciou que o everolimus é capaz de remover macrófagos de placas ateroscleróticas desses animais por meio de autofagia, um mecanismo novo e também dependente da inibição da mTOR. Se comprovadas tais propriedades em seres humanos, esse fármaco tornar-se-á uma atrativa opção na abordagem das placas vulneráveis, que, embora muitas vezes não apresentem estenoses críticas, trazem consigo iminente risco de ruptura e desencadeamento de quadros coronários agudos.

\section{Polímero de flúor}

Apesar de classificado como durável, esse polímero possui propriedades biocompatíveis, sendo seu uso consagrado em máquinas de hemodiálise, enxertos vasculares, suturas cardíacas, etc.

Além disso, sua baixa espessura $(<7,8 \mu \mathrm{m})$ aliada à flexibilidade favorece a manutenção de sua integridade ao ultrapassar lesões críticas em artérias bastante tortuosas/calcificadas.

No que tange à liberação do everolimus, estudos in vitro demonstraram que $25 \%$ do fármaco são liberados nas primeiras 24 horas do procedimento. Ao final do primeiro mês, $75 \%$ do fármaco já foram liberados. Os $25 \%$ restantes são completamente eliminados até o quarto mês da intervenção (Figura 2).

A Figura 3 compara as principais características estruturais entre o stent Xience ${ }^{\mathrm{TM}} \mathrm{V}$ e os outros três SF aprovados pelo Food and Drug Administration (FDA): Cypher $^{\mathrm{TM}}$, Taxus ${ }^{\mathrm{TM}}$ e Endeavor ${ }^{\mathrm{TM}}$.

\section{ESTUDOS CLÍNICOS}

\section{SPIRIT I (First-in-Man)}

Esse estudo representa a primeira avaliação do stent $X_{i e n c e}{ }^{T M} V$ em seres humanos, e foi conduzido em nove centros europeus, entre dezembro de 2003 e abril de 2004.

Trata-se de um estudo randomizado que incluiu 60 pacientes $\left(28\right.$ tratados com $\mathrm{Xience}^{\mathrm{TM}} \mathrm{V}$ e 32 com seu equivalente não-farmacológico, o stent Multi-Link 


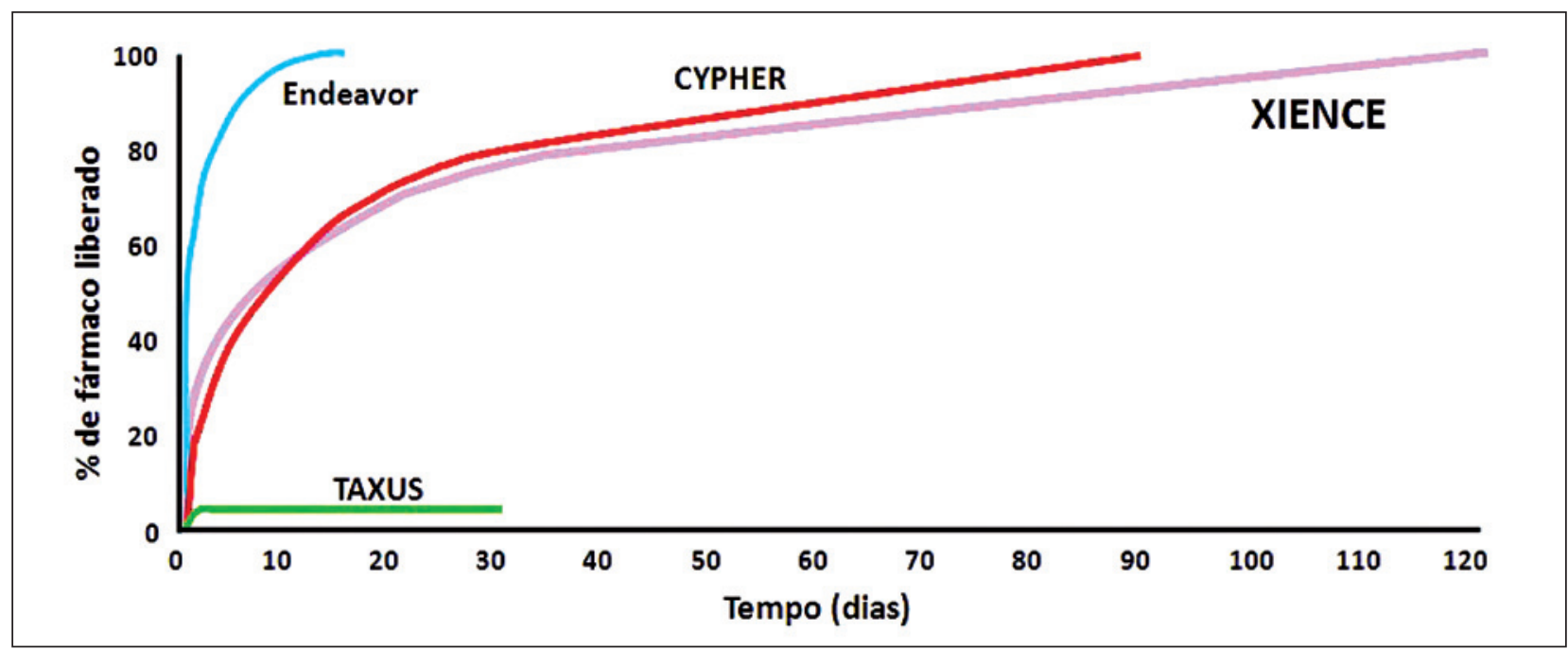

Figura 2 - Curva de eluição do everolimus no stent Xience ${ }^{T M} \vee$ versus os principais stents farmacológicos aprovados para uso clínico. Notase que esse novo stent leva mais tempo (quatro meses) para liberar completamente seu fármaco antiproliferativo.

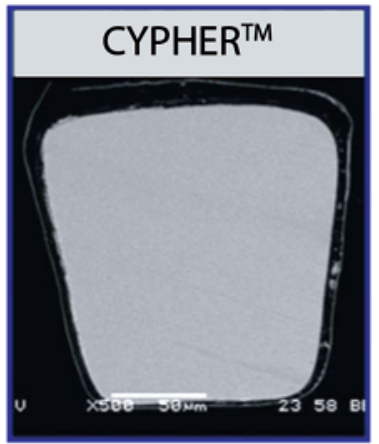

Espessura das hastes

$140 \mu \mathrm{m}$

Espessura das polímero

$13.7 \mu \mathrm{m}$

Quantidade de fármaco

$150 \mu \mathrm{g} / 18 \mathrm{~mm}$

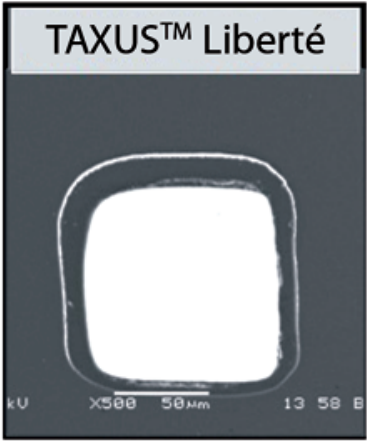

Espessura das hastes

$97 \mu \mathrm{m}$

Espessura das polímero

$17.8 \mu \mathrm{m}$

Quantidade de fármaco

$100 \mu \mathrm{g} / 16 \mathrm{~mm}$

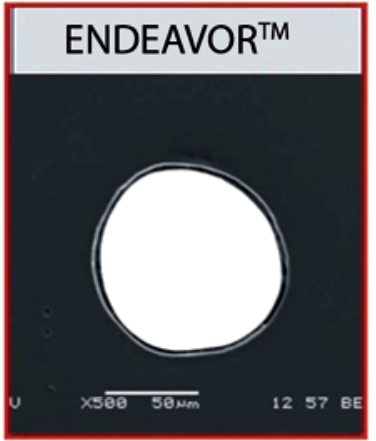

Espessura das hastes

$91 \mu \mathrm{m}$

Espessura das polímero

$4.8 \mu \mathrm{m}$

Quantidade de fármaco

$180 \mu \mathrm{g} / 18 \mathrm{~mm}$

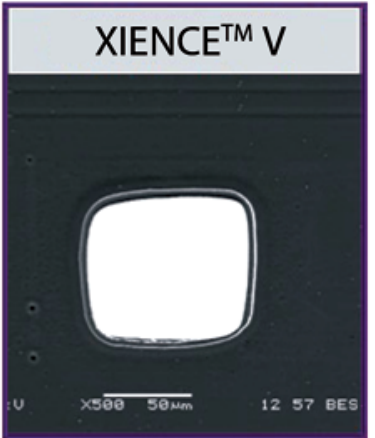

Espessura das hastes

$81 \mu \mathrm{m}$

Espessura das polímero

$7.8 \mu \mathrm{m}$

Quantidade de fármaco

$88 \mu \mathrm{g} / 18 \mathrm{~mm}$

Figura 3 - Características estruturais dos quatro principais stents farmacológicos aprovados para uso clínico. O stent Xience ${ }^{\mathrm{TM}} \mathrm{V}$ destaca-se pelo seu menor perfil e quantidade de fármaco eluído.

Vision $^{\mathrm{TM}}$ ) portadores de lesões coronárias de baixa complexidade (lesões únicas, de novo, em vasos nativos passíveis de tratamento com stent 3,0 mm x $18 \mathrm{~mm}$ ). O objetivo primário desta avaliação foi comparar a perda luminal tardia e o diâmetro mínimo da luz no interior dos stents no reestudo angiográfico de seis meses. A terapia antiplaquetária dupla foi mantida por apenas três meses. As principais características angiográficas do procedimento, bem como os resultados da angiografia quantitativa e ultra-sonografia aos seis meses, estão dispostos na Tabela 1.

Notavelmente, o SF com everolimus reduziu marcantemente a formação neo-intimal no interior do stent, fato demonstrado pela sua significante superioridade sobre o stent $\mathrm{Vision}^{\mathrm{TM}}$ no que diz respeito a redução da perda tardia, porcentagem de estenose e porcentagem de obstrução do stent ao final de seis meses $^{14}$. 
TABELA 1

Principais características angiográficas e ultra-sonográficas dos estudos do Programa Xience $^{\mathrm{TM}} \mathrm{V}$ (procedimento/primeiro reestudo angiográfico)

\begin{tabular}{|c|c|c|c|c|c|c|}
\hline & \multirow[b]{2}{*}{ Rand } & \multirow[b]{2}{*}{$\begin{array}{l}\text { Número de } \\
\text { pacientes }\end{array}$} & \multicolumn{4}{|c|}{ Procedimento índice } \\
\hline & & & DRV, mm & $\begin{array}{l}\text { Extensão da } \\
\text { lesão, mm }\end{array}$ & $\begin{array}{c}\% \text { DE } \\
\text { (pós-implante), mm }\end{array}$ & $\begin{array}{c}\text { DLM } \\
\text { (pós-implante), mm }\end{array}$ \\
\hline SPIRIT I** & Sim & 50 & $2,61 \pm 0,40$ & $10,1 \pm 2,6$ & $12,0 \pm 5,0$ & $2,38 \pm 0,25$ \\
\hline - EES & $(1: 1)$ & 23 & $2,71 \pm 0,28$ & $10,9 \pm 3,3$ & $15,0 \pm 6,0$ & $2,45 \pm 0,31$ \\
\hline - MLV & & 27 & & & & \\
\hline SPIRIT II & Sim & 299 & $2,70 \pm 0,52^{*}$ & $13,0 \pm 5,7$ & $13,0 \pm 6,0$ & $2,49 \pm 0,40^{*}$ \\
\hline - EES & $(3: 1)$ & 222 & $2,82 \pm 0,58^{*}$ & $13,2 \pm 6,4$ & $13,0 \pm 6,0$ & $2,62 \pm 0,45^{*}$ \\
\hline - PES & & 77 & & & & \\
\hline SPIRIT III*** & Sim & 436 & $2,77 \pm 0,45$ & $14,7 \pm 5,6$ & $0,3 \pm 8,9$ & $2,71 \pm 0,43$ \\
\hline - EES & $(2: 1)$ & 302 & $2,76 \pm 0,46$ & $14,7 \pm 5,7$ & $-0,2 \pm 9,9$ & $2,74 \pm 0,41$ \\
\hline - PES & & 134 & & & & \\
\hline
\end{tabular}

$\mathrm{DRV}=$ diâmetro de referência do vaso; $\mathrm{DE}=$ diâmetro de estenose; $\mathrm{DLM}=$ diâmetro luminal mínimo; $\mathrm{EES}=\mathrm{Xience}$ TM $\mathrm{V}$; $\mathrm{MLV}=\mathrm{Multi}-$ link Vision ${ }^{\mathrm{TM}} \mathrm{N} / \mathrm{D}=$ informação não disponível; PES = Taxus ${ }^{\mathrm{TM}}$. * Valor de $\mathrm{p}<0,05$. ** Do total de 60 pacientes incluídos no estudo SPIRIT I, apenas 50 apresentaram análise angiográfica seriada. *** Do total de 1.002 pacientes incluídos no estudo SPIRIT III, somente 436 submeteram-se a reestudo angiográfico seriado.

TABELA 1

Principais características angiográficas e ultra-sonográficas dos estudos do Programa Xience ${ }^{\mathrm{TM}} \mathrm{V}$ (procedimento/primeiro reestudo angiográfico) (continuação)

\begin{tabular}{|c|c|c|c|c|c|c|}
\hline & \multirow[b]{2}{*}{$\begin{array}{l}\text { Meses até } \\
\text { o reestudo }\end{array}$} & \multicolumn{5}{|c|}{ Seguimento angiográfico/ultra-sonográfico } \\
\hline & & $\begin{array}{c}\% \mathrm{DE} \\
\mathrm{mm}\end{array}$ & $\begin{array}{c}\text { DLM, } \\
\text { mm }\end{array}$ & $\begin{array}{c}\text { Perda tardia, } \\
\text { mm }\end{array}$ & $\begin{array}{l}\text { Reestenose } \\
\text { binária (\%) }\end{array}$ & $\begin{array}{c}\text { \% de obstrução } \\
\text { do stent }\end{array}$ \\
\hline SPIRIT I** & & $16,0 \pm 8,0$ & $2,28 \pm 0,33^{*}$ & $0,1 \pm 0,21$ & $0^{*}$ & $8,0 \pm 10,4^{*}$ \\
\hline \multicolumn{2}{|l|}{ - EES } & & & & & \\
\hline \multicolumn{2}{|l|}{$-\mathrm{MLV}$} & $39,0 \pm 14$ & $1,58 \pm 0,41^{*}$ & $0,87 \pm 0,37$ & $25,9 *$ & $28,1 \pm 14^{*}$ \\
\hline \multicolumn{2}{|l|}{ SPIRIT II } & $14,7 \pm 7,0^{*}$ & $2,38 \pm 0,50$ & $0,12 \pm 0,29 *$ & 1,3 & $2,5 \pm 4,7^{*}$ \\
\hline - EES & 6 & & & & & \\
\hline \multicolumn{2}{|l|}{ - PES } & $36,7 \pm 18,1^{*}$ & $2,27 \pm 0,55$ & $0,37 \pm 0,38^{*}$ & 3,5 & $7,4 \pm 7,0^{*}$ \\
\hline \multicolumn{2}{|l|}{ SPIRIT III ${ }^{* * *}$} & $5,9 \pm 16,4^{*}$ & $2,56 \pm 0,53$ & $0,16 \pm 0,41^{*}$ & 2,3 & 6,9 \\
\hline - EES & 8 & & & & & \\
\hline \multicolumn{2}{|l|}{ - PES } & $10,3 \pm 21,4^{*}$ & $2,45 \pm 0,65$ & $0,30 \pm 0,53^{*}$ & 5,7 & 11,2 \\
\hline \multicolumn{7}{|c|}{ 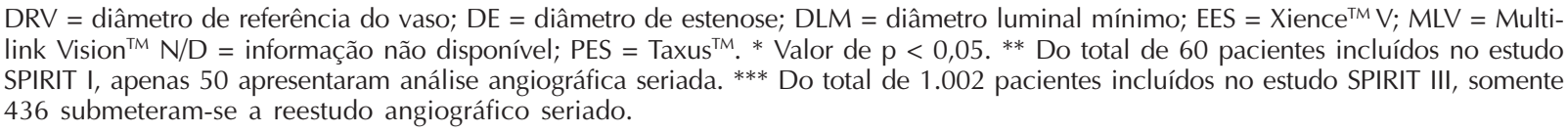 } \\
\hline
\end{tabular}

Ao final de um ano de seguimento, eventos cardíacos adversos maiores (ECAM) foram observados em $15,4 \%$ dos pacientes tratados com SF e em 21,4\% dos que receberam stents não-farmacológicos (SNF) $(p=0,102)^{15}$. A angiografia e a ultra-sonografia realizadas naquela ocasião evidenciaram aumento da perda luminal tardia e da porcentagem de obstrução do stent em relação aos seis meses (perda luminal de $0,24 \mathrm{~mm}$ $\pm 027 \mathrm{~mm}$ vs. $0,10 \mathrm{~mm} \pm 0,21 \mathrm{~mm}$ aos seis meses e porcentagem de obstrução do stent de 10\% $\pm 7,0 \%$ vs. $8,0 \% \pm 10,4 \%$, porém em níveis ainda bastante abaixo dos SNF e compatíveis com outros SF disponíveis.

Recentemente publicados, os resultados clínicos de dois anos praticamente não se alteraram em relação aos de 12 meses (ECAM de 21,4\% no grupo SF vs. $25 \%$ no grupo controle, p > 0,05). Notavelmente, até esse período, não foram descritos casos de trombose de stent em ambos os grupos ${ }^{16}$. 


\section{SPIRIT II}

Diante dos entusiasmadores resultados do estudo FIM, decidiu-se por continuar a avaliação desse novo instrumental, comparando-o a outro SF, em uma coorte maior e mais complexa.

O estudo SPIRIT II avaliou de maneira randomizada (3:1) o SF Xience ${ }^{T M} V(n=223$ pacientes $)$ versus $\mathrm{o}$ também SF Taxus ${ }^{\mathrm{TM}}$ ( $\mathrm{n}=77$ pacientes) em portadores de até duas lesões coronárias de novo em vasos de 2,5 $\mathrm{mm}$ a 3,75 $\mathrm{mm}$ e extensão $<28 \mathrm{~mm}$. O objetivo primário dessa análise era demonstrar a não-inferioridade do stent Xience ${ }^{\mathrm{TM}}$ em reduzir perda luminal tardia no interior do stent aos seis meses.

As principais características angiográficas da população incluída, bem como os resultados angiográficos e ultra-sonográficos de seis meses, encontram-se na Tabela 1.

Nesse estudo, o stent $\mathrm{Xience}^{\mathrm{TM}} \mathrm{V}$ apresentou resultados tão bons que não só se mostrou nãoinferior como também exibiu superioridade em relação ao Taxus ${ }^{\mathrm{TM}}$ em reduzir a perda tardia $(0,12 \mathrm{~mm}$ vs. 0,37 mm, p 0,001 para não-inferioridade e superioridade) ${ }^{17}$. No entanto, dado o limitado tamanho da população avaliada, esse benefício angiográfico não resultou em superioridade clínica, com ambos os stents apresentando equivalentes taxas de ECAM $(2,7 \%$ no grupo Xience ${ }^{T M} V$ vs. 5,0\% no Taxus $\left.{ }^{\text {TM }} ; p>0,05\right)$. Também não foram evidenciadas diferenças significativas nas taxas de reestenose binária $(1,3 \%$ vs. 3,5\%) e revascularização da lesão-alvo (1,8\% vs. 2,0\%) entre os dois grupos ( $p=$ NS para ambas as comparações).

Ao final de doze meses de seguimento clínico, o grupo tratado com Xience ${ }^{\mathrm{TM}} \mathrm{V}$ apresentou taxa de ECAM de $2,7 \%$ versus $9,2 \%$ no grupo $\operatorname{Taxus}^{\mathrm{TM}}(p=0,04)$, sobretudo pela maior necessidade de nova revascularização da lesão-alvo entre os pacientes que receberam SF com paclitaxel $\left(5,3 \%\right.$ vs. 1,8\%) ${ }^{18}$. É importante ressaltar que as taxas de trombose de stent foram baixas nos dois grupos $\left(0,5 \%\right.$ no grupo $\mathrm{Xience}^{\mathrm{TM}} \mathrm{V}$ vs. $1,3 \%$ no grupo Taxus ${ }^{\mathrm{TM}} ; \mathrm{p}=\mathrm{NS}$ ), com apenas um caso de trombose tardia no grupo que recebeu SF com everolimus. Nesse estudo, a terapia antiplaquetária dupla foi mantida por seis meses.

\section{SPIRIT III}

Uma vez demonstrada a superioridade do stent Xience $^{T M} V$ sobre o stent Taxus ${ }^{T M}$ por meio de objetivos angiográficos e ultra-sonográficos, era hora de avançar para uma segunda etapa, tendo desfechos clínicos como objetivos primários da comparação, visando ao estabelecimento do real impacto desse SF de segunda geração no cenário prático da cardiologia intervencionista.

Um total de 1.002 pacientes foram randomizados em 65 centros norte-americanos para receber $\mathrm{Xience}^{\mathrm{TM}}$
$V$ ( $n=669$ pacientes) ou Taxus ${ }^{\text {TM }}$ (333 pacientes). Os critérios de inclusão e exclusão assemelharam-se aos do estudo SPIRIT II e a terapia antiplaquetária dupla foi também mantida por seis meses para ambos os grupos.

No reestudo angiográfico de oito meses, o SF com everolimus mostrou-se superior ao SF com paclitaxel em reduzir a perda luminal tardia e a reestenose binária $^{19}$ (Tabela 1). Do ponto de vista clínico, o stent Xience $^{\mathrm{TM}} \mathrm{V}$ mostrou-se superior em reduzir a taxa de ECAM combinada aos nove meses $(4,6 \%$ vs. $8,1 \%$; risco relativo [RR] 0,56, intervalo de confiança de $95 \%$ [IC 95\%] 0,34-0,94; p = 0,03) e um ano (6,0\% vs. 10,3\%; RR 0,58, IC 95\% 0,37-0,90; p = 0,02), pela menor quantidade de infarto e nova revascularização da lesão-alvo entre os pacientes que receberam esses stents.

No que se refere à trombose das endopróteses, as taxas de assemelharam entre os grupos $(1,7 \%$ na coorte tratada com Xience $\mathrm{TM}^{\mathrm{TM}} \mathrm{V}$ vs. $1,3 \%$ no grupo Taxus $\left.{ }^{\mathrm{TM}} ; p=0,78\right)$, sendo apenas $0,3 \%$ delas após os primeiros 30 dias no grupo que recebeu o SF de segunda geração (no grupo controle houve 0,6\% de trombose após o primeiro mês do procedimento).

Recentemente apresentados por Stone et al. (EuroPCR 2008, Barcelona, Espanha) ${ }^{20}$, os resultados clínicos de dois anos confirmam a superioridade do stent $\mathrm{Xience}^{\mathrm{TM}}$ $\checkmark$ sobre o Taxus ${ }^{\text {TM }}$ em reduzir ECAM combinados $(7,3 \%$ vs. 12,8\%; RR 0,55; IC 95\% 0,36-0,83; p = 0,004), principalmente pela redução da necessidade de reintervenção na lesão-alvo (4,3\% vs. 6,9\%; RR 0,60, IC 95\% 0,35-1,04; $p=0,07)$. Notou-se, porém, incremento na taxa de trombose em ambos os grupos, com o surgimento do primeiro caso de trombose muito tardia (> 360 dias) entre os pacientes tratados com esse SF de segunda geração (trombose tardia de $0,3 \%$ no grupo Xience $^{\mathrm{TM}} \mathrm{V}$ vs. 1,0\% no grupo Taxus $\left.{ }^{\mathrm{TM}} ; \mathrm{p}=0,34\right)$.

\section{Análise combinada dos bancos de dados dos estudos SPIRIT II e III}

Visando ao aumento da consistência dos dados do programa SPIRIT, Stone et al. apresentaram, no EuroPCR de $2007^{21}$, os resultados clínicos e angiográficos de uma metanálise envolvendo os pacientes incluídos nos estudos SPIRIT II e III. Favoreceu a execução dessa análise o fato de os autores terem acesso aos bancos de dados originais de cada estudo e, sobretudo, o fato de os critérios de inclusão e exclusão, os stents comparados e a terapêutica antiplaquetária adotada serem os mesmos nos dois estudos.

Com isso, um total de 1.302 pacientes foi incluído (802 tratados com Xience ${ }^{\text {TM }} \mathrm{V}$ e 410 com Taxus $^{\text {TM }}$ ). A análise angiográfica quantitativa demonstrou redução de $58 \%$ na perda luminal tardia no interior do stent e de $61 \%$ na taxa de reestenose binária no grupo tratado com o SF de segunda geração. 
A Figura 4 apresenta a comparação dos desfechos clínicos entre os dois grupos. Observa-se redução de $50 \%$ na taxa de ECAM entre os pacientes tratados com os stent $\mathrm{Xience}^{\mathrm{TM}} \mathrm{V}$, o que se deveu basicamente à redução na necessidade de nova intervenção na lesão-alvo (2,4\% vs. 5,1\%; RR 0,47, IC 95\% 0,26-0,87; $\mathrm{p}=0,01)$. Na análise dos desfechos clínicos de segurança (óbito, infarto agudo do miocárdio e trombose), não se observou diferença entre os stents até o período avaliado.

\section{NOVOS ESTUDOS E PERSPECTIVAS}

Encontram-se em fase de recrutamento de pacientes três grandes ensaios clínicos com o SF Xience ${ }^{\mathrm{TM}} \mathrm{V}$ : SPIRIT IV, SPIRIT V e SPIRIT Women.

O estudo SPIRIT IV pretende avaliar, de forma randomizada versus o stent Taxus ${ }^{\mathrm{TM}}$, a eficácia e a segurança do stent $\mathrm{Xience}^{\mathrm{TM}} \mathrm{V}$ no tratamento de pacientes multiarteriais (até três lesões por paciente). Esse estudo está sendo conduzido nos Estados Unidos e incluirá 1.125 pacientes com lesões de novo.

Por sua vez, o estudo SPIRIT $\vee$ pretende comparar o stent Xience ${ }^{T M} \mathrm{~V}$ ao stent Taxus ${ }^{T M}$ no tratamento de pacientes com diabetes melito. Esse estudo contará também com um registro, em paralelo, apenas com pacientes diabéticos tratados com Xience ${ }^{\mathrm{TM}} \mathrm{V}$. Enquanto o estudo controlado prevê a inclusão de 300 indivíduos, o registro almeja avaliar 2.700 diabéticos.

Por fim, o SPIRIT Women representa o primeiro estudo na cardiologia intervencionista com foco exclusivo em mulheres com doença arterial coronária. Esse estudo, da mesma forma que o SPIRIT $V$, contará com um subgrupo randomizado (vs. Cypher ${ }^{\mathrm{TM}}$ ) e outro apenas com registro de mulheres tratadas com esse novo SF.

\section{CONCLUSÕES}

A despeito dos entusiasmadores resultados apresentados pelo SF $X$ ience $^{\mathrm{TM}} \mathrm{V}$ no âmbito dos ensaios clínicos controlados, ainda não se dispõe de dados suficientes a respeito da segurança e da eficácia desse novo SF para o tratamento de populações nãoselecionadas (de "mundo real").

Espera-se que agora, com sua aprovação pelas principais agências reguladoras mundiais (incluindo o FDA, nos Estados Unidos), seu uso mais generalizado confirme os bons resultados até então obtidos.

\section{REFERÊNCIAS BIBLIOGRÁFICAS}

1. Morice MC, Serruys PW, Sousa JE, Fajadet J, Ban Hayashi E, Perin M, et al; RAVEL Study Group. Randomized study with the sirolimus-coated bx velocity balloon-expandable stent in the treatment of patients with de novo native coronary artery lesions. A randomized comparison of a sirolimus-eluting stent with a standard stent for coronary revascularization. N Engl J Med. 2002;346(23):1773-80.

2. Moses JW, Leon MB, Popma JJ, Fitzgerald PJ, Holmes DR, O'Shaughnessy C, et al; SIRIUS Investigators. Sirolimuseluting stents versus standard stents in patients with stenosis in a native coronary artery. N Engl J Med. 2003;349(14): 1315-23.

3. Stone GW, Ellis SG, Cox DA, Hermiller J, O'Shaughnessy C, Mann JT, et al; TAXUS-IV Investigators. A polymer-based, paclitaxel-eluting stent in patients with coronary artery disease. N Engl J Med. 2004;350(3):221-31.

4. Sousa JE, Costa MA, Tuzcu EM, Yadav JS, Ellis S. New frontiers in interventional cardiology. Circulation. 2005;111(5): 671-81.

5. McFadden EP, Stabile E, Regar E, Cheneau E, Ong AT, Kinnaird $T$, et al. Late thrombosis in drug-eluting coronary stents after discontinuation of antiplatelet therapy. Lancet. 2004,364(9444):1519-21.

6. Feres F, Costa JR Jr, Abizaid A. Very late thrombosis after drugeluting stents. Catheter Cardiovasc Interv. 2006;68(1):83-8.

7. Biondi-Zoccai GG, Lotrionte $M$, Agostoni P, Abbate A, Fusaro $M$, Burzotta $F$, et al. A systematic review and meta-

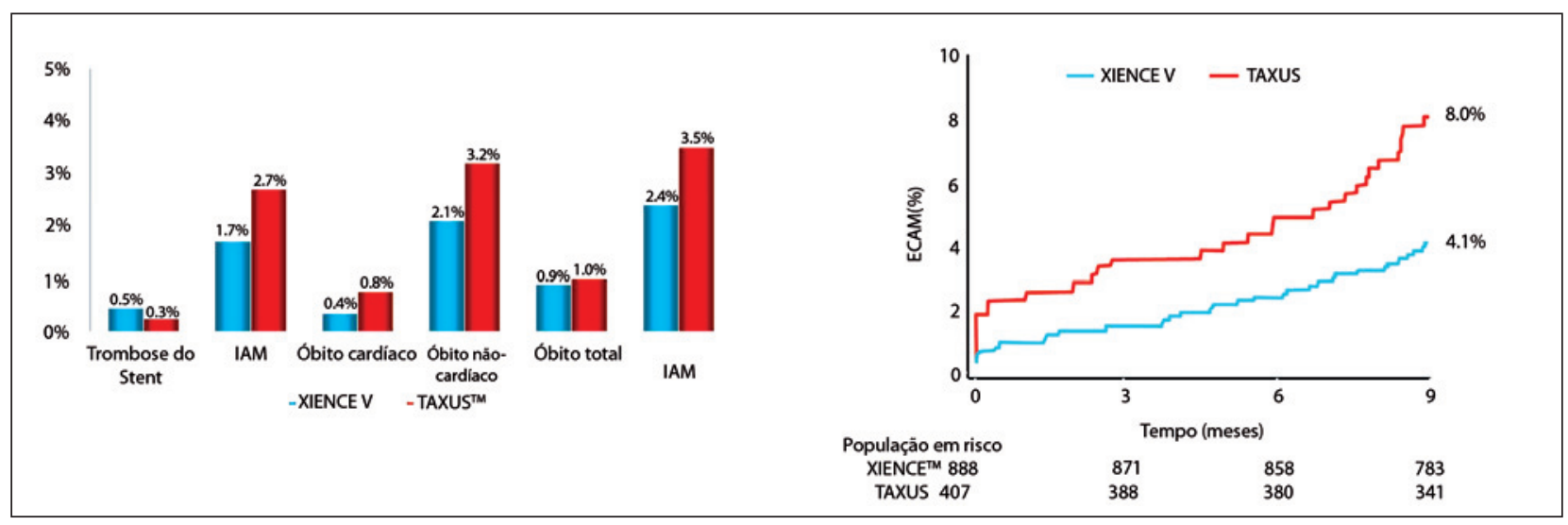

Figura 4 - Desfechos clínicos dos pacientes incluídos na metanálise dos estudos controlados SPIRIT II e III. No painel à esquerda, observase equivalência entre os stents farmacológicos $X_{i e n c e}{ }^{T M} \mathrm{~V}$ e Taxus ${ }^{T M}$ no quesito segurança até o período de nove meses dessa análise. No que se refere à redução de eventos cardíacos maiores (painel à direita), o stent farmacológico de segunda geração mostrou-se superior. ECAM = eventos cardíacos adversos maiores; IAM = infarto agudo do miocárdio. 
analysis on the hazards of discontinuing or not adhering to aspirin among 50,279 patients at risk for coronary artery disease. Eur Heart J. 2006;27(22):2667-74.

8. Nebeker JR, Virmani R, Bennett CL, Hoffman JM, Samore $\mathrm{MH}$, Alvarez J, et al. Hypersensitivity cases associated with drug-eluting coronary stents: a review of available cases from the Research on Adverse Drug Events and Reports (RADAR) project. J Am Coll Cardiol. 2006;47(1):175-81.

9. Joner M, Finn AV, Farb A, Mont EK, Kolodgie FD, Ladich $E$, et al. Pathology of drug-eluting stents in humans: delayed healing and late thrombotic risk. J Am Coll Cardiol. 2006; 48(1):193-202.

10. Siqueira DA, Abizaid AA, Costa JR, Feres F, Mattos LA, Staico $R$, et al. Late incomplete apposition after drug-eluting stent implantation: incidence and potential for adverse clinical outcomes. Eur Heart J. 2007;28(11):1304-9.

11. Hagemeister J, Baer FM, Schwinger RH, Höpp HW. Compliance of a cobalt chromium coronary stent alloy: the COVIS trial. Curr Control Trials Cardiovasc Med. 2005;6:17.

12. Carter AJ. TOR of the cell cycle: Are there important implications for diabetics in the era of the drug-eluting stent? Catheter Cardiovasc Interv. 2004;61(2):233-6.

13. Verheye S, Martinet W, Kockx MM, Knaapen MW, Salu K, Timmermans JP, et al. Selective clearance of macrophages in atherosclerotic plaques by autophagy. J Am Coll Cardiol. 2007;49(6):706-15.

14. Serruys PW, Ong A, Piek JJ, Neumann FJ, van der Giessen WJ, Wiemer $\mathrm{M}$, et al. A randomized comparison of a durable polymer everolimus-eluting stent with a bare metal coronary stent: the SPIRIT first trial. Eurolntervention. 2005;1(1):58-65.

15. Tsuchida K, Piek JJ, Neumann FJ, van der Giessen WJ, Wiemer M, Zeiher A, et al. One-year clinical results of a durable polymer everolimus-eluting stent in de novo coronary narrowings (The SPIRIT First Trial). Eurolntervention. 2005; $1: 266-72$.

16. Beijk M, Neumann FJ, Wiemer M, Grube E, Haase J, Thuesen $\mathrm{L}$, et al. Two-year results of a durable polymer everolimuseluting stent in de novo coronary artery stenosis (The SPIRIT FIRST Trial). Eurolntervention. 2007;3:206-12.

17. Serruys PW, Ruygrok P, Neuzner J, Piek J, Seth A, Schofer $\mathrm{J}$, et al. A randomised comparison of an everolimus-eluting coronary stent with a paclitaxel-eluting coronary stent: the SPIRIT II trial. Eurolntervention. 2006;2:286-94.

18. Ruygrok P, Desaga M, van den Branden F, Rasmussen K, Suryapranata $H$, Dorange $C$, et al., on behalf of the SPIRIT II Investigators. One year clinical follow-up of the XIENCE $\checkmark$ Everolimus-eluting stent system in the treatment of patients with de novo native coronary artery lesions: the SPIRIT II study. Eurolntervention. 2007;3:315-20.

19. Stone GW, Midei M, Newman W, Sanz M, Hermiller JB, Williams J, et al.; SPIRIT III Investigators. Comparison of an everolimus-eluting stent and a paclitaxel-eluting stent in patients with coronary artery disease: a randomized trial. JAMA. 2008;299(16):1903-13.

20. Stone GW. In: EuroPCR 2008; Barcelona, Espanha; 2008. 21. Stone GW. In: EuroPCR 2007; Barcelona, Espanha; 2007. 